\title{
Introduction: Special Issue on Families and Disabilities
}

\author{
Jennifer Haskin \\ Heather Dillaway
}

Wayne State University

\begin{abstract}
Articles featured in Volume 17 of Michigan Family Review focus on issues faced by families when a member, or members, have disabilities. Family researchers of disability have often focused on how physical (visible) disability affects family life and family wellbeing. In this special issue, we offer a selection of articles that highlight cognitive (often invisible) disabilities and mental health, such as Post Traumatic Stress Disorder (PTSD), Dementia due to aging, learning disabilities, and child abuse.

Shildrick (2002) and other disability scholars suggest that we should think of a "disability" as an "impairment." That is, broadly defined, a disability is typically something that impedes one's ability to navigate their social world and relationships, or participate fully in society. This impairment or impediment could be physical, cognitive, or emotional. Typically we think of disability as related to the physical body first and foremost but disability scholars remind us that cognitive and/or mental disabilities affect individuals as much as any physical impairment can. Social scientists and disability advocates further refer to disabilities as socially constructed. This is not to say that disabilities do not have a biological, physiological, neurological, or physical reality but, rather, that "neither impairment nor disability can be defined in purely biomedical terms, because social arrangements and expectations make essential contributions to impairment and disability" (Wendell 1996: 57). In other words, when it comes to disabilities, the biological and the social work together in the creation and understanding of disability, as well as who should be responsible for the care of the disabled or impaired. This is how disability or impairment becomes a "family" issue.

Societies like the United States tend to relegate members of society who are defined as having a disability (or disabilities) to the private realm - that is, the realm of the family. Disability, by default, then becomes a personal and family "problem." When disabilities cause individuals to be unable to fulfill their basic needs, our first instinct is to expect family members to provide special care. When there are barriers to disabled individuals' health care, paid work, or mobility, it is also often family members who are expected to pick up the slack. When disabled individuals experience barriers or constraints to their participation in society in any form, or any difficulty at all in living their daily lives, they may also take out their frustrations on or share these feelings with family members. Thus, families deal with the consequences of the barriers and constraints that disabled individuals may face. When disabled individuals experience physical or emotional
\end{abstract}


symptoms of their impairments, it is often family members who must negotiate these symptoms. When impaired individuals need or want an advocate, it also often family members who step to their aid. Familial units are called upon, expected to be there for, and negotiate disability and impairment on a daily basis.

Ideas within the literature on disability studies can be useful in expanding how we conceptualize familial experiences and think about how families negotiate disability or impairment. Disability scholars teach us to 'separat[e] out "impairment" (that is, the functional limitations of our bodies and minds, or the actual physical or cognitive "disabilities") from the subjectivity of "disability" (that is, the attitudes towards disability or the structural barriers that a disabled person might face in society) (Morris, 2001, p. 2). Hughes and Patterson (1997) and Thomas (1999) further remind us that we need to pay attention to the embodied experiences of impairment, while also paying attention to the social barriers, new relations and interactions, and personal experiences associated with physical or cognitive impairment. As Thomas (1999) explains, impairment is not a fixed property of a person but, rather, a 'social relational' entity, which means that how one lives with a physical or cognitive impairment is more telling about what an impairment truly is for a person than any material reality of the body or mind (Shildrick, 2002). We can take this further by examining how impairment as a social relational entity - can affect family experience. For instance, PTSD becomes real on a daily basis when it affects one's ability to talk to one's children or argue with one's intimate partner. Learning disabilities become real when parents realize they must interact in particular ways with diagnosed children, and histories of abuse become real when they affect individuals' willingness to engage in caregiving relationships later on. Dementia is lived by family caregivers perhaps as much as it is lived by the impaired person, just perhaps in a different way.

The articles in this issue of Michigan Family Review touch on just some of the ways that cognitive (and often invisible) disabilities affect family members and family experiences. For example, Rosanne Dizazzo-Miller and her colleagues describe the lived experiences of family members who are caregivers of persons with dementia, and how caregiver needs vary depending on the stage of dementia. Michelle Pryor-Kowalski discusses the ways in which parenting styles and parental reactions to a child's learning disability may impact delinquent behavior in adolescents. Terri Kovach uses qualitative phenomenological analysis to describe how Post Traumatic Stress Disorder (PTSD) as a result of combat trauma shapes family relationships for Vietnam veterans. Heleana Thexois' conceptual piece applies a socio-moral perspective to investigate the demands placed on adult children when it comes time to provide care for their aging parents, and proposes that those caregiving decisions and relationships may be even more complicated when there is a history of child abuse.

When reading each of these articles readers are asked to expand their conceptualizations of both disability itself and how disability impacts family life. Dizazzo-Miller et al. suggest that caregiving is not static and that caregivers need different supports at different times, as elderly parents' dementia is not static either. Pryor-Kowalski highlights how much the parent-child relationship may be 
altered with the diagnosis and experience of a learning disability, and how parents must figure out how to address and navigate this disability in their children's tweens and teens. She finds that parenting style and parent-child interactions may have an effect on how a learning disabled child comes into contact with and behaves in other social institutions, and especially whether a learning disabled child becomes criminally delinquent. Parents are often constantly negotiating what the learning disability means for their family as well as what it means for their children's schooling. Kovach shows how PTSD, as an impairment, can affect veterans' abilities to participate in or maintain close familial relationships, navigate familial situations, and manage their fears within family settings. Kovach suggests that there are important implications of PTSD for families across the life course, as PTSD may not lessen in later life. Adult children and long-time spouses must interact with older veterans who may still have the same PTSD symptoms 40 and 50 years later. Vietnam veterans who have lost contact with family members must now contemplate who may take care of them in older age. While we might not always think of PTSD as a disability, it is an impairment that affects families for an entire lifetime. Finally, Thexois brings us back to the issue of elderly caregiving and asks us to think about the moral obligations that adult children may feel when negotiating their parents' cognitive decline. She also asks us to contemplate whether and how those moral-ethical obligations to care for parents may be different in cases where children suffered from abuse. In this case, we might think of child abuse as a form of impairment or disability as well, one that can affect how individuals deal with family relations (or any other set of relations) over time. Disability can represent a multitude of statuses and experiences, and can affect family life and family relationships in many different ways over time. Much more research is needed about the connections and overlaps in the experiences of disabilities and families.

We hope that this special issue helps readers challenge their own ideas about what disability means and how it might affect families. While we are only able to offer a sample of articles here, the authors push us to contemplate cognitive as well as physical impairments, invisible as well as visible disabilities, the changing nature of (and response to) disability across the life course, and the various familial relationships and obligations that might be affected and challenged by the presence of disability. We also hope that these articles push readers to think about doing their own research on families and disabilities, for our knowledge is still very partial on these topics.

We would like to thank all of the dedicated peer reviewers and authors for contributing to this special issue of Michigan Family Review. It is truly a community of scholars who creates a special issue like this.

\section{References}

Hughes, B. \& Patterson, K. (1997). The social model of disability and the disappearing body: Towards a sociology of impairment. Disability \& Society, 12 (3), 325-40. 
Morris, J. (2001) Impairment and disability: Constructing an ethics of care that promotes human rights. Hypatia, 16 (4), 1-16.

Shildrick, M. (2002) Embodying the monster: Encounters with the vulnerable self. London: Sage Publications.

Thomas, C. (1999). Female forms: Experiencing and understanding disability. Buckingham: Open University Press.

Wendell, S. (1996). The rejected body: Feminist philosophical reflections on disability. New York: Routledge. 